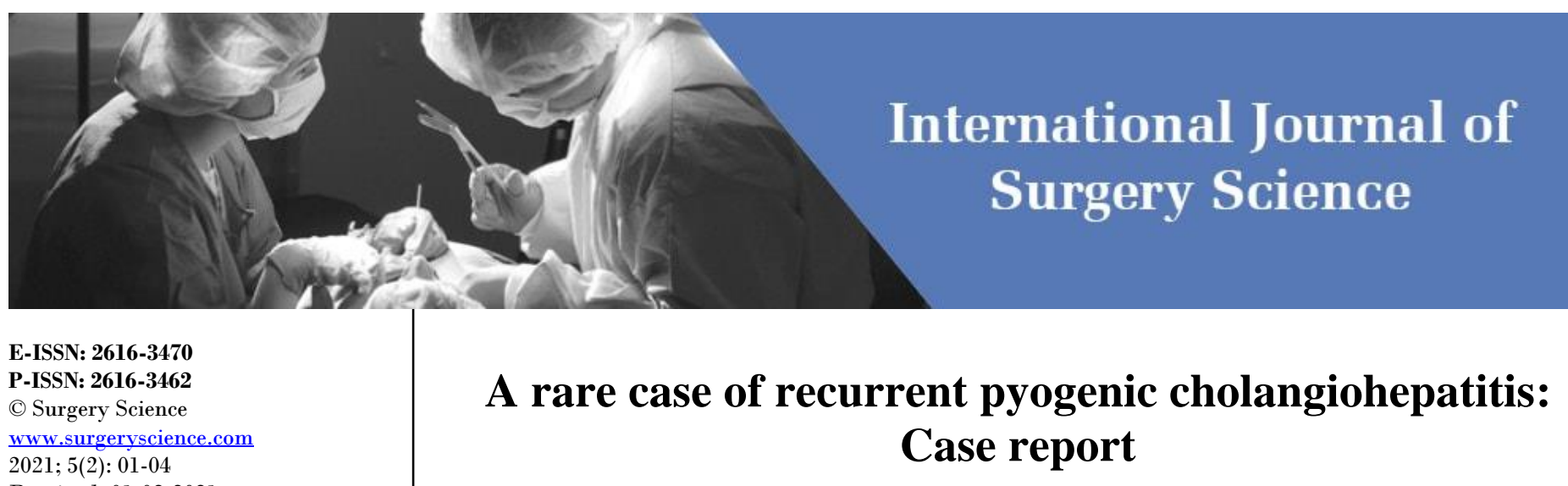

Received: 01-02-2021

Accepted: 04-03-2021

Dr. B Santhi

Professor and Head of the Department, Department of General Surgery, Government Kilpauk Medical College and Hospital, Chennai, Tamil Nadu, India

\section{Dr. Sugumar}

Professor and Head of the

Department, Department of Surgical Gastroenterology, Government Kilpauk Medical College and Hospital, Chennai, Tamil Nadu, India

\section{Dr. Sridevi}

Assistant Professor, Department of General Surgery, Kilpauk Medical College and Hospital, Chennai,

Tamil Nadu, India

\section{Dr. Uma}

Assistant Professor, Department of General Surgery, Government Kilpauk Medical College and Hospital, Chennai, Tamil Nadu, India

\section{Dr. Ramprasadh}

Assistant Professor, Department of General Surgery, Government Kilpauk Medical College and Hospital, Chennai, Tamil Nadu, India

\section{Dr. Nandhini V}

Post Graduate Student,

Department of General Surgery, Government Kilpauk Medical

College and Hospital, Chennai, Tamil Nadu, India

\section{Corresponding Author:}

\section{Dr. B Santhi}

Professor and Head of the Department, Department of General Surgery, Government Kilpauk Medical College and Hospital, Chennai, Tamil Nadu, India

\section{Dr. B Santhi, Dr. Sugumar, Dr. Karthikeyan Sridevi, Dr. Uma, Dr. Ramprasath and Dr. Nandhini V}

DOI: https://doi.org/10.33545/surgery.2021.v5.i2a.650

\section{Abstract}

A 47-year-old female presented with Easy fatigability, abdominal pain for 4 days. Past history of open cholecystectomy with Common bile duct exploration and Choledocho Duodenostomy done 13 years before. She later had multiple episodes of abdomen pain with fever on and off. ERCP revealed stone in common bile duct but not retrieved and patient lost follow-up. During this hospitalisation patient had fever, Jaundice and Sepsis with Acute Kidney Injury. Ultrasound Abdomen showed Hypoechoic lesion in seg IV left lobe liver. Mild pneumobilia. CECT Abdomen showed Pneumobilia, ill-defined hypodense lesions in segment IVA, II, III causing left lobe Intrahepatic biliary radicle dilation. MRCP showed Multiple tiny T2 hyperintense lesions with surrounding edema in SEGMENT IVB? Cholangitic abscess. Patient was taken up for surgery. Intraoperatively Left lateral segment II, III fibrotic, left hepatic duct \& branches grossly dilated, stones retrieved from LHD; Left lateral sectionectomy, hepaticojejunostomy, jejunojejunostomy with access loop done. This was a rare case of recurrent pyogenic cholangiohepatitis managed surgically.

Keywords: common bile duct exploration, Choledocho duodenostomy, ERCP, retained stone, intrahepatic biliary radicle dilatation, cholangitic abscess

\section{Introduction}

RPC is due to repeated primary bacterial infections of the biliary system and the development of pigmented stones, ductal strictures. Both intrahepatic, extrahepatic bile ducts are involved.

Management involves the removal of stones, establishing adequate biliary drainage, and respecting the non-functioning segments of the liver that serve as foci of infection. Due to the risk of cholangiocarcinoma cases with associated liver atrophy, liver abscesses, third-order ductal calculi are best treated with partial hepatectomy.

\section{Case Report}

A 47-year-old female with Easy fatigability and loss of appetite and abdominal pain for 4 days with No h/o abdominal distension, vomiting, fever, pale stools, high coloured urine at present H/O open cholecystectomy with CBD exploration done 13yrs before, Choledocho Duodenostomy done in 2006, the patient had sump syndrome ERCP done in 2010, stone in CBD could not be retrieved. O/E - pallor \& icterus present Per abdomen- right subcostal incision scar+ tenderness in epigastric and right hypochondrial region Initial blood investigations severe microcytic hypochromic anemia with neutrophilic leukocytosis severe thrombocytopenia Total bilirubin was elevated predominantly direct bilirubin, liver enzymes normal Urea/Creatinine elevated - Acute kidney injury.

Hemoglobin -8.1gm\% Total Count-26000 Platelet-29000 Patient total bilirubin-5.2 direct bilirubin - 3.4 SGOT/PT/ALP- 88/88/166 Urea/Creatinine- 140/2.8

The patient later developed fever, which was high grade intermittent associated with chills, rigor. Her urine output decreased, and she was treated with IV Fluids, higher antibiotics and stabilized. Serial RFT monitoring was done. The patient recovered from Acute kidney injury. IgM ELISA Dengue Negative Malarial Parasite /Micro Filariae Negative PT INR-normal. 


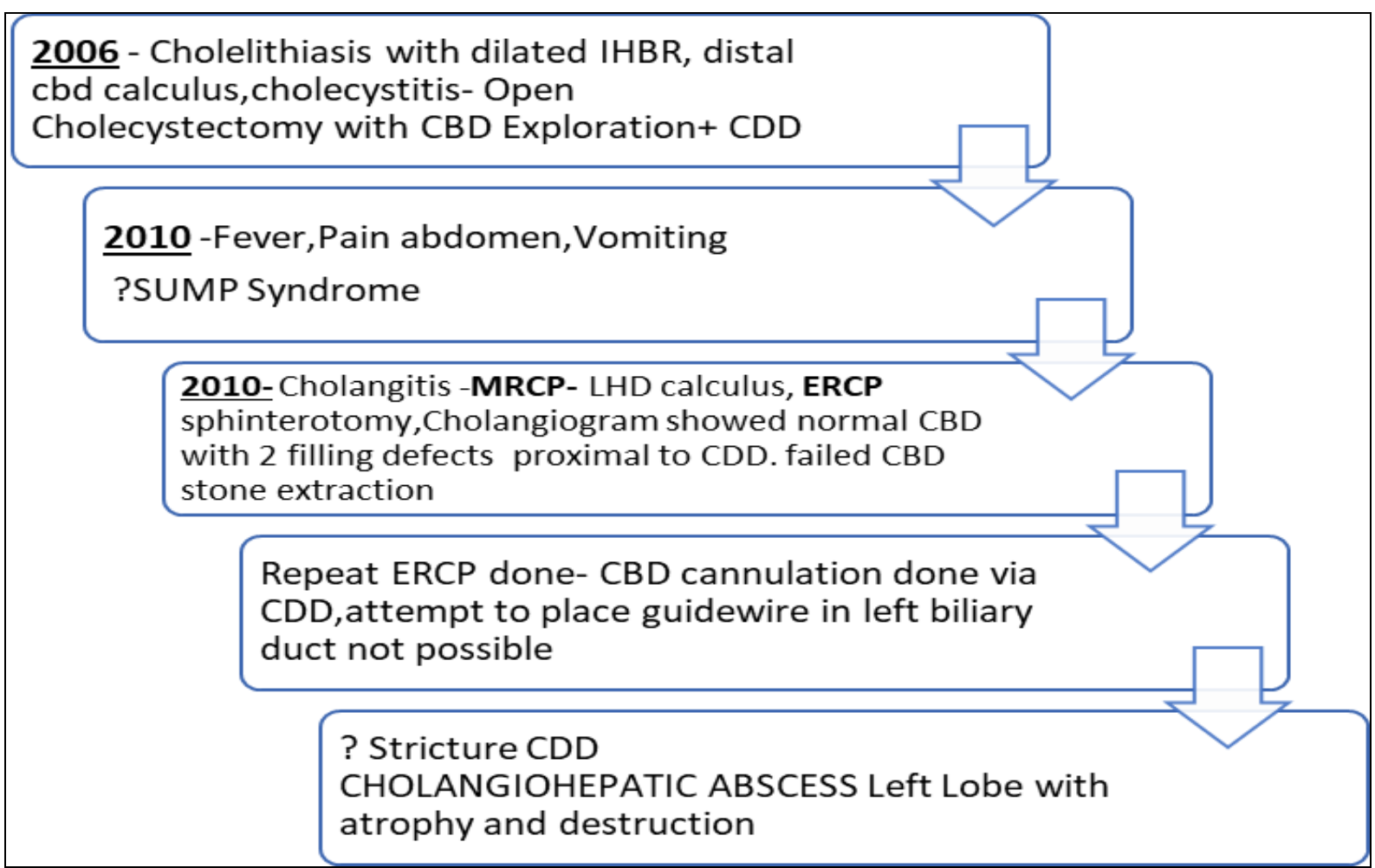

Fig 1: Case History

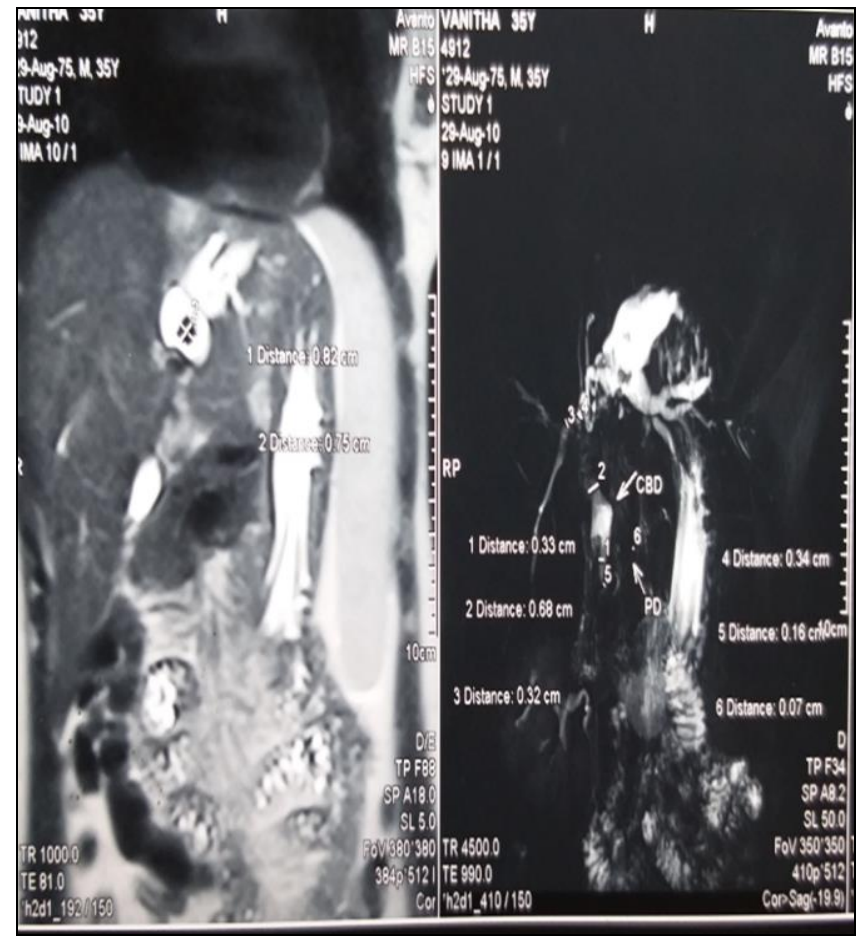

Fig 2: MRCP in 2010

MRCP shows Intra Hepatic Biliary Radicle dilatation in the left side and left hepatic duct dilated Left Hepatic Duct calculi causing biliary obstruction. CHD and CBD prominent. No CBD calculus.

\section{ERCP Findings}

Bow sphincterotomy done, Cholangiogram showed normal CBD with 2 filling defects proximal to CDD, Balloon trolling done, CBD Stone not extracted.

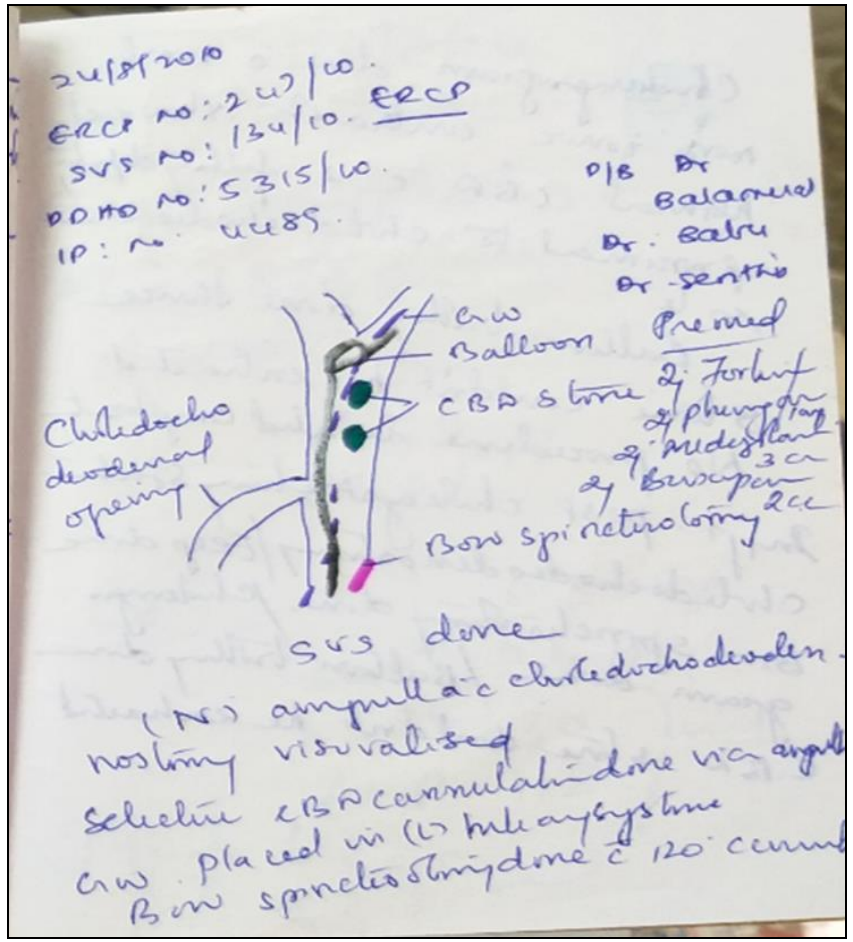

Fig 3: ERCP in 2010

Investigations

USG abdomen showed IHBR grossly dilated in the left lobe of the liver. $3.8 * 5 \mathrm{~cm}$ hypoechoic lesion noted in seg IV in left lobe liver. Mild pneumobilia, post-cholecystectomy status, and Splenomegaly. CECT Abdomen showed pneumobilia, illdefined hypodense lesions $5.4 * 5.5 \mathrm{~cm}$ in segment IVA, II, III causing left lobe IHBR dilation. 

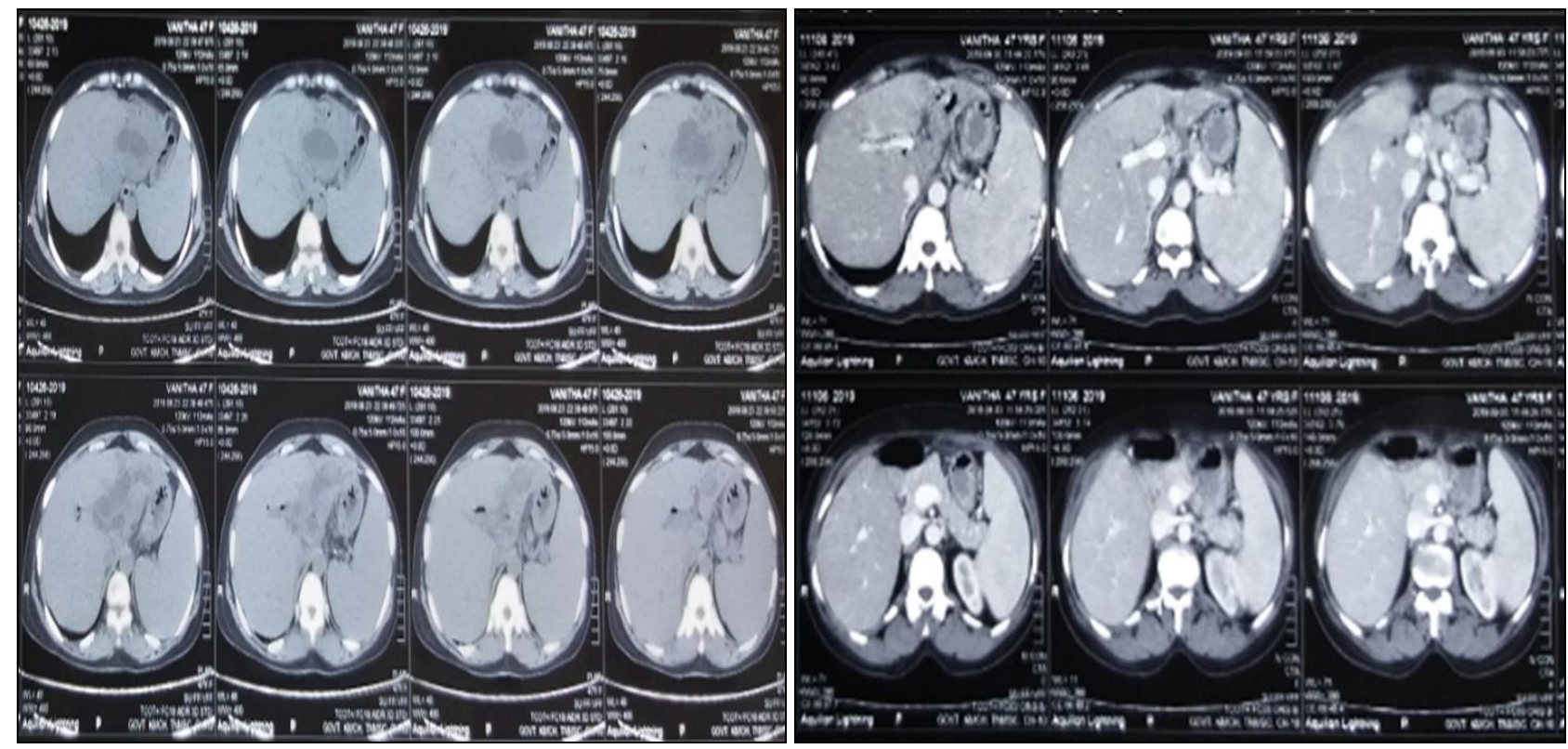

Fig 4: CECT Abdomen

Portal venous Doppler was Normal. Medical gastroenterologist opinion obtained. They suspected intrahepatic cholangiocarcinoma. Tumour markers- CEA, CA19-9 were found to be normal.

Oesophago -Gastro Duodenoscopy findings - Esophagus fundus, body, antrum- normal, Pseudodiverticulum seen in D1 D2 Junction. The fistulous opening is seen in the inferior wall of D1, D2 junction MRCP showed Pneumobilia with IHBR dilated in the left lobe liver, altered signal intensity noted in left lobe of the liver. Multiple tiny T2 hyperintense lesions with surrounding edema in SEGMENT IVB $2.5 * 2.2 \mathrm{~cm}$ ? Cholangitic abscess. T2 Hyperintense collection with air fluid level noted in GB fossa $3.9 * 3 \mathrm{~cm}$ with fistulous communication to proximal $1 / 3 \mathrm{rd}$. of $\mathrm{CBD}$ and D1.

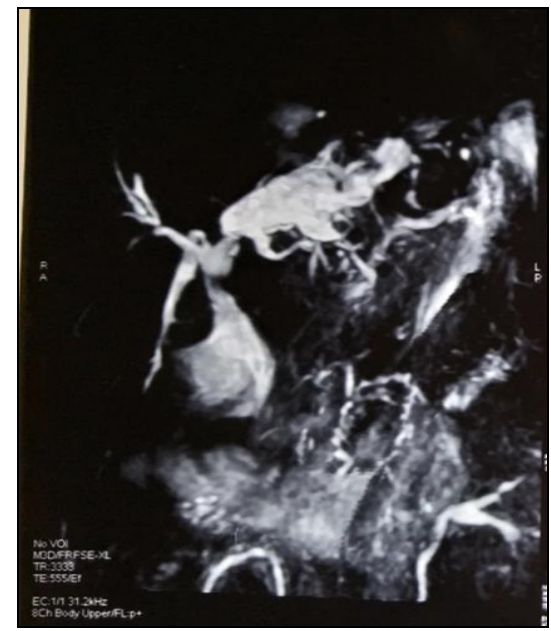

Fig 5: MRCP Abdomen

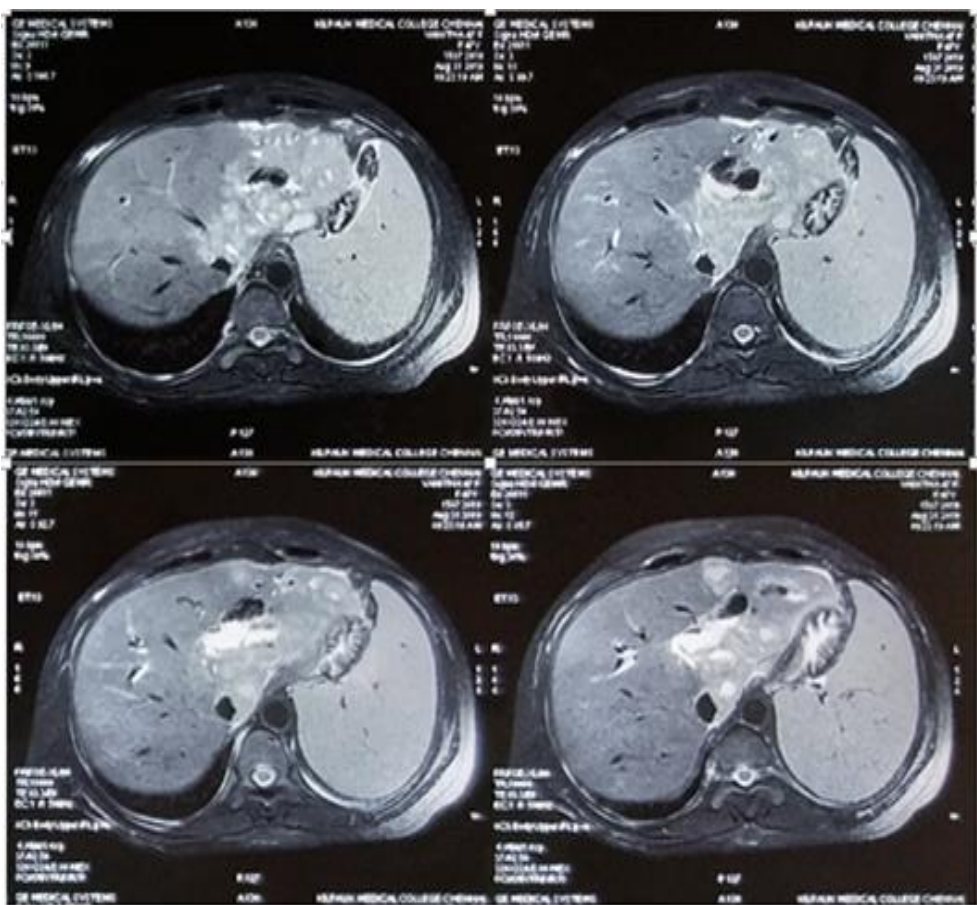

Fig 6: MRCP Abdomen 
The patient diagnosed with cholangiohepatitis with ? fibrosis of left lateral hepatic lobes and was planned for surgery.

\section{Surgery}

Under general anesthesia, a rooftop incision was made, and the abdomen opened in layers.

\section{Following Intraoperative findings noted}

1. Left lateral segment II, III fibrotic

2. Left hepatic duct \& branches grossly dilated

3. Duodenum pylorus densely adherent to the liver, the liver adherent to parietal wall

Resection of choledochoduodenostomy, choledochectomy done. Left lateral sectionectomy done, branches of the left hepatic vein, hepatic artery, portal vein ligated \& cut. Common hepatic duct divided, Infant feeding tube passed and flushed with saline, few stones retrieved. Hepaticojejunostomy done (End to Side). Grossly dilated LHD on the cut surface of liver closed in 2 layers. Jejunojejunostomy done and an access loop placed as hepaticocutaneous jejunostomy.

The patient recovered and was discharged on the 7 th postoperative day. The patient is under regular follow-up now.

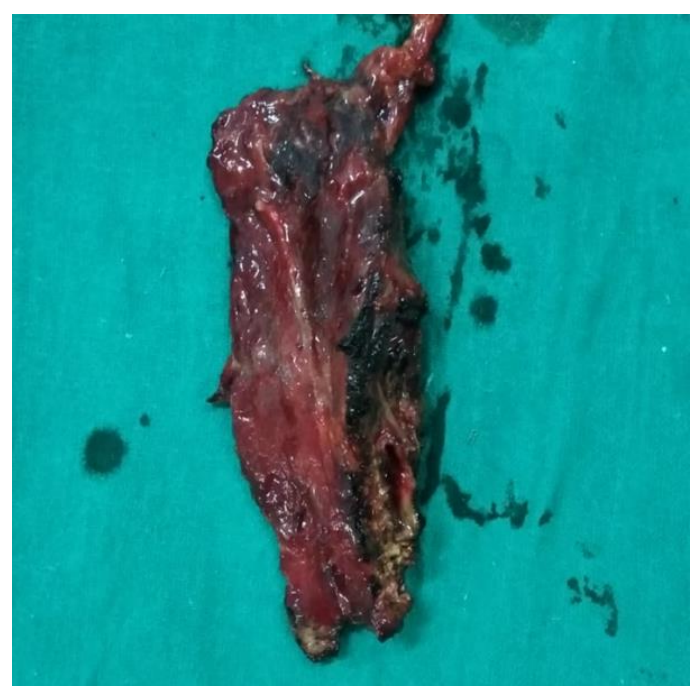

Fig 7: Left Lateral Sectionectomy

Histopathology report: Portion of liver $9 \times 4 \times 2 \mathrm{~cm}$ congested surface, cut surface grey white, normal liver parenchyma couldnot be made out. Microscopic section shows liver parenchyma with plenty of dilated sinusoids marked congestion, minimal inflammation around portal triad and dense fibrosis. A small portion of adherent bileduct surrounded by chronic inflammatory cells and nerve bundles are seen. Features suggestive of chronic inflammatory pathology.

\section{Discussion}

RPC is also known as Oriental cholangiohepatitis. The repeated or severe infection leads to transmural inflammation of the ducts resulting in stenosis in the larger ducts and smaller peripheral ducts, showing more tubular narrowing. As a result of obstruction, together with parenchymal damage to the adjoining liver, the rest of the ducts dilate. The Bacteria induce overexpression of gel-forming apomucin (MUC2 and MUC5AC) in biliary epithelial cells through synthesis of TNF- $\alpha$ protein kinase C. Results in Mucin hypersecretion and Calculi formation - calcium bilirubinate stones. Followed by the establishment of infection by bowel microorganisms in the small biliary radicles. Organisms may enter the portal vein during the serious attack of infection. Infection begins in cholangioles of portal triads, the hepatocytes show vacuolation, and may undergo necrosis and fibrosis of intrahepatic ducts leading to cholangitic liver abscess formation ${ }^{[1]}$.

Conservative treatment fails in $30 \%$ of pts in an acute attack. Complications occur in $35 \%$ of pts require emergency surgical/endoscopic/radiological procedures like Choledochotomy t tube drainage, Transhepatic drain tubes, Cholecystectomy Hepatic resection, Transduodenal sphinteroplasty, Electrohydraulic lithotripsy, Emergency ERCP Endoscopic decompression/percutaneous transhepatic drainage $[1,2]$.

Definitive surgeries include Cholecystectomy, CBD exploration, hepatectomy, partial hepatectomy, hepaticocutaneous jejunostomy, stricturoplasty, $\quad$ Supradudenal choledochoduodenostomy. Due to the risk of cholangiocarcinoma cases with associated liver atrophy, liver abscesses, third-order ductal calculi are best treated with partial hepatectomy ${ }^{[3]}$.

Follow-up: The long-term result is best measured by the reappearance of stones and strictures after 5 years of follow-up and by the occurrence of portal hypertension, bleeding esophageal varices, ascites, liver failure, and cholangiocarcinoma. Access loop gives us repeated access to the biliary tract by choledochoscopy, if there is a recurrence of stones, strictures ${ }^{[1,4]}$.

\section{Conclusion}

In our case, the Recurrent Pyogenic cholangiohepatitis is due to retained left hepatic duct stone which leads to repeated infections culminating in cholangiohepatitis with fibrosis of the left lateral segment. Hence patients with retained stones after ERCP either in CBD or proximal ducts should be constantly kept in follow-up and all measures should be done either to retrieve it and prevent repeat infections.

\section{References}

1. Jarnagin WR, Belghiti J, Blumgart LH. Blumgart's surgery of the liver, biliary tract, and pancreas. Edn Elsevier Saunders, Philadelphia 2012;6(1):725-741.

2. Townsend, Jr Courtney M, Daniel Beauchamp R, Mark Evers B, Kenneth L. Mattox. Sabiston Textbook of Surgery. Edn Elsevier - Health Sciences Division, Philadelphia, 2016;20(1):1454-1455

3. Verweij KE, Van Buuren H. Oriental cholangiohepatitis (recurrent pyogenic cholangitis): A case series from the Netherlands and brief review of the literature. The Netherlands journal of medicine 2016;74(9):401-405.

4. Cheon YK, Cho YD, Moon JH, Lee JS, Shim CS. Evaluation of long-term results and recurrent factors after operative and nonoperative treatment for hepatolithiasis. Surgery 2009;146(5):843-853. doi: 10.1016/j.surg.2009.04.009 